\title{
ON DANKWERTS' TRANSFORMATION FOR TWO VARIABLE COUPLED SYSTEMS
}

\author{
James M. Hill and Alex McNabB
}

The problem of obtaining explicit solutions to coupled linear reaction-diffusion partial differential equations is generally recognised as technically very difficult. Frequently it is possible to deduce seemingly simple expressions for Laplace or Fourier transforms of the solution but such transforms tend not to be amenable to simple inversion and usually involve, for example, square roots within a square root. Fortunately however, a general uncoupling procedure has previously been established which provides explicit integral expressions in terms of classical heat functions. Such expressions are especially useful for problems with zero boundary data but non-zero initial data. The purpose of this paper is to provide the formal details necessary to deduce corresponding uncoupling transformations for two dependent variables, which preserve zero initial data and constant boundary data. For the case of one dependent variable such a transformation is known as Dankwerts' transformation. For coupled systems the existence of a Dankwerts' transformation means that together with the existing uncoupling transformation, solutions of boundary value problems involving constant boundary data, can be decomposed, just as for the single heat equation, into a contribution from the initial condition and zero boundary data and a contribution from non-zero boundary data and zero initial condition. The problem considered is highly non-trivial and the final expressions obtained are correspondingly complicated. Nevertheless the end results are explicit and together with standard integration routines constitute a powerful solution procedure.

\section{INTRODUCTION}

Multi-component systems undergoing diffusion and chemical reactions frequently give rise to coupled systems of linear differential equations involving several dependent variables. For example, for two diffusing species undergoing first-order chemical reactions we have the following coupled system for the concentrations $c_{j}(\underset{\sim}{\boldsymbol{x}}, t)(j=1,2)$

$$
\begin{aligned}
& \frac{\partial c_{1}}{\partial t}=d_{1} \nabla^{2} c_{1}+a_{11} c_{1}+a_{12} c_{2} \\
& \frac{\partial c_{2}}{\partial t}=d_{2} \nabla^{2} c_{2}+a_{21} c_{1}+a_{22} c_{2}
\end{aligned}
$$

Received 23 May 1989

Copyright Clearance Centre, Inc. Serial-fee code: 0004-9729/90 \$A2.00+0.00. 
where $\nabla^{2}$ denotes the usual spatial Laplacian, the diffusivities $d_{j}(j=1,2)$ are positive constants which we suppose are such that $d_{1}>d_{2}$ and the matrix $A=\left[a_{i j}\right]$ is assumed here to be a general constant matrix. More generally, Hill [2] shows that the coupled equations

$$
\begin{aligned}
& \frac{\partial c_{1}}{\partial t}=d_{1} L\left(c_{1}\right)+a_{11} c_{1}+a_{12} c_{2}, \\
& \frac{\partial c_{2}}{\partial t}=d_{2} L\left(c_{2}\right)+a_{21} c_{1}+a_{22} c_{2},
\end{aligned}
$$

where $L$ is any linear spatial differential operator, admit formal solutions of the form,

$$
\begin{aligned}
& c_{1}(\underset{\sim}{x}, t)=e^{a_{11} t} h_{1}\left(\underset{\sim}{x}, d_{1} t\right) \\
& +\frac{e^{\lambda t}}{\left(d_{1}-d_{2}\right)} \int_{d_{2} t}^{d_{1} t} e^{-\mu \xi}\left\{\left(\frac{a_{12} a_{21}\left(\xi-d_{2} t\right)}{\left(d_{1} t-\xi\right)}\right)^{1 / 2} I_{1}(\eta) h_{1}(\underset{\sim}{x}, \xi)\right. \\
& \left.\quad+a_{12} I_{0}(\eta) h_{2}(\underset{\sim}{x}, \xi)\right\} d \xi, \\
& c_{2}(\underset{\sim}{x}, t)=e^{a_{22} t} h_{2}\left(\underset{\sim}{x}, d_{2} t\right) \\
& +\frac{e^{\lambda t}}{\left(d_{1}-d_{2}\right)} \int_{d_{2} t}^{d_{1} t} e^{-\mu \xi}\left\{\left(\frac{a_{12} a_{21}\left(d_{1} t-\xi\right)}{\left(\xi-d_{2} t\right)}\right)^{1 / 2} I_{1}(\eta) h_{2}(\underset{\sim}{x}, \xi)\right. \\
& \left.\quad+a_{21} I_{0}(\eta) h_{1}(\underset{\sim}{x}, \xi)\right\} d \xi,
\end{aligned}
$$

where the constants $\lambda$ and $\mu$ are given by

$$
\lambda=\left(\frac{d_{1} a_{22}-d_{2} a_{11}}{d_{1}-d_{2}}\right), \mu=\left(\frac{a_{22}-a_{11}}{d_{1}-d_{2}}\right),
$$

and $I_{0}$ and $I_{1}$ are modified Bessel functions with $\eta$ defined by

$$
\eta=\frac{2\left(a_{12} a_{21}\right)^{1 / 2}}{\left(d_{1}-d_{2}\right)}\left[\left(d_{1} t-\xi\right)\left(\xi-d_{2} t\right)\right]^{1 / 2}
$$

Further $h_{1}(\underset{\sim}{x}, t)$ and $h_{2}(\underset{\sim}{x}, t)$ are understood to be solutions of

$$
\frac{\partial h}{\partial t}=L(h)
$$

satisfying the same initial conditions as $c_{1}(\underset{\sim}{x}, t)$ and $c_{2} \underset{\sim}{\underset{\sim}{x}, t)}$ respectively. This result is important because, for example for coupled reaction-diffusion equations, certain 
boundary value problems can be reduced to similar boundary value problems but for the classical diffusion equation (see Hill [2]). Now for a single reaction-diffusion equation

$$
\frac{\partial c}{\partial t}=d L(c)-a c
$$

with zero initial condition and prescribed as constant on the boundary, the Dankwerts' transformation (see for example Crank [1])

$$
c(x, t)=e^{-a t} h(x, d t)+\frac{a}{d} \int_{0}^{d t} e^{-a \xi / d} h(x, \xi) d \xi
$$

is well-known to preserve the zero initial and constant boundary condition for $h(x, t)$ and moreover $h(x, t)$ satisfies (1.6). The purpose of this paper is to present formulae for a Dankwerts' transformation for a coupled system of the form (1.1) or (1.2). That is, we seek uncoupling transformations which preserve zero initial conditions and constant boundary conditions.

In the following section we briefly describe the general matrix structure of uncoupling transformations and present the basic equations for the determination of the kernel matrix. The general abstract structure of the Dankwerts' transformation is described elsewhere (McNabb [6]) and the purpose of this paper, for two dependent variables, is to provide the formal mathematical details to derive an explicit representation which might be suitable for use in a specific problem. The formal details are given in Section 3 and 4 and the final expressions are summarised in Section 5.

\section{GENERAL MATRIX STRUCTURE OF UNCOUPLING TRANSFORMATIONS}

In abstract terms the essential matrix structure of the uncoupling transformation (1.3) is identified in $\mathrm{McNabb}$ [5] for any number of dependent variables. In particular McNabb [5] shows that solutions of the coupled system

$$
\frac{\partial \underset{\sim}{c}}{\partial t}=D L(\underset{\sim}{c})+\underset{\sim}{c}
$$

where $D=\left[d_{j}\right]$ is a positive constant diagonal matrix and $A=\left[a_{i j}\right]$ is a general matrix not involving spatial variables, can be expressed in terms of solutions of the uncoupled system

$$
\frac{\partial \underset{\sim}{H}}{\partial t}=D L(\underset{\sim}{H})
$$


by means of relations of the form,

$$
\begin{aligned}
\underset{\sim}{c}(\underset{\sim}{x}, t) & =(J+K) \underset{\sim}{H}(\underset{\sim}{x}, t) \\
& \left.=\underset{\sim}{J}(\underset{\sim}{x}, t)+\int_{0}^{t} k^{+}(t, s) \underset{\sim}{H}(\underset{\sim}{x}, s) d s+\int_{t}^{\infty} k^{-}(t, s) \underset{\sim}{H} \underset{\sim}{x, s}\right) d s
\end{aligned}
$$

where $k(t, x)=k^{+}(t, s)$ for $s<t$ and $k(t, s)=k^{-}(t, s)$ for $s>t$. If for simplicity we assume at the outset firstly that the elements of $D$ are distinct and the variables are labelled such that

$$
d_{1}>d_{2}>d_{3} \ldots d_{n}>0
$$

and secondly that the matrix $A$ is a constant matrix with all elements non-zero then in these circumstances the matrix $J$ in (2.3) is diagonal and given simply by

$$
J(t)=\left[e^{a_{j j} t}\right]
$$

Further the kernel matrices $k^{+}(t, s)$ and $k^{-}(t, s)$ are obtained as solutions of the hyperbolic equations

$$
\begin{aligned}
& \frac{\partial k^{+}}{\partial t} D+D \frac{\partial k^{+}}{\partial s}=A k^{+} D, 0 \leqslant t \leqslant \infty, 0 \leqslant s \leqslant t \\
& \frac{\partial k^{-}}{\partial t} D+D \frac{\partial k^{-}}{\partial s}=A k^{-} D, 0 \leqslant s \leqslant \infty, 0 \leqslant t \leqslant s
\end{aligned}
$$

with boundary conditions

$$
k^{+}(t, 0)=k^{-}(0, s)=0
$$

and jump conditions along $s=t$,

$$
\left[k^{+}(t, t)-k^{-}(t, t)\right] D-D\left[k^{+}(t, t)-k^{-}(t, t)\right]=\left(A-A^{0}\right) J D,
$$

where with the above assumptions $A^{0}$ is simply the diagonal matrix $\left[a_{j j}\right]$. The unsolved problem of obtaining explicit closed form solutions of $(2.6)-(2.8)$ for more than two dependent variables is discussed at length in the survey paper Hill and McNabb [3].

Also, in a recent article McNabb [6] shows that the generalised uncoupling Dankwerts' transformation, with kernel matrix $k^{*}(t, s)$, satisfies precisely the above equations (2.6) - (2.8) except that $(2.7)_{1}$ is replaced by

$$
k^{*+}(t, 0)=-D^{-1} A D .
$$


The purpose of this paper is to present the details for the determination of $k^{*}(t, s)$ for the case of two dependent variables only. For further details on uncoupling transformations we refer the reader to Hill and McNabb [3] and McNabb [5, 6].

Explicitly in component form and for two dependent variables the first column of the kernel matrix $k^{*}(t, s)$ satisfies

$$
\begin{aligned}
\frac{\partial k_{11}^{*}}{\partial t}+\frac{\partial k_{11}^{*}}{\partial s} & =a_{11} k_{11}^{*}+a_{12} k_{21}^{*}, \\
\frac{\partial k_{21}^{*}}{\partial t}+\frac{d_{2}}{d_{1}} \frac{\partial k_{21}^{*}}{\partial s} & =a_{21} k_{11}^{*}+a_{22} k_{21}^{*},
\end{aligned}
$$

with boundary conditions

$$
\begin{gathered}
k_{11}^{*-}(0, s)=k_{21}^{*-}(0, s)=0, \\
k_{11}^{*+}(t, 0)=-a_{11}, k_{21}^{*+}(t, 0)=-a_{21} d_{1} / d_{2} .
\end{gathered}
$$

As described in Hill and McNabb [3] the general rules for solving such systems are firstly that components of $k^{*}(t, s)$ are continuous across characteristics except their own and secondly that all components suffer a jump across the characteristic $s=t$. With these rules in mind we observe from (2.11) $)_{1,2}$ and Figure 1 that $k_{11}^{*-}(t, s)$ and $k_{21}^{*-}(t, s)$ are both identically zero in the region EOC and moreover $k_{21}^{*}$ suffers a jump across $s=t$ of magnitude (determined from (2.8))

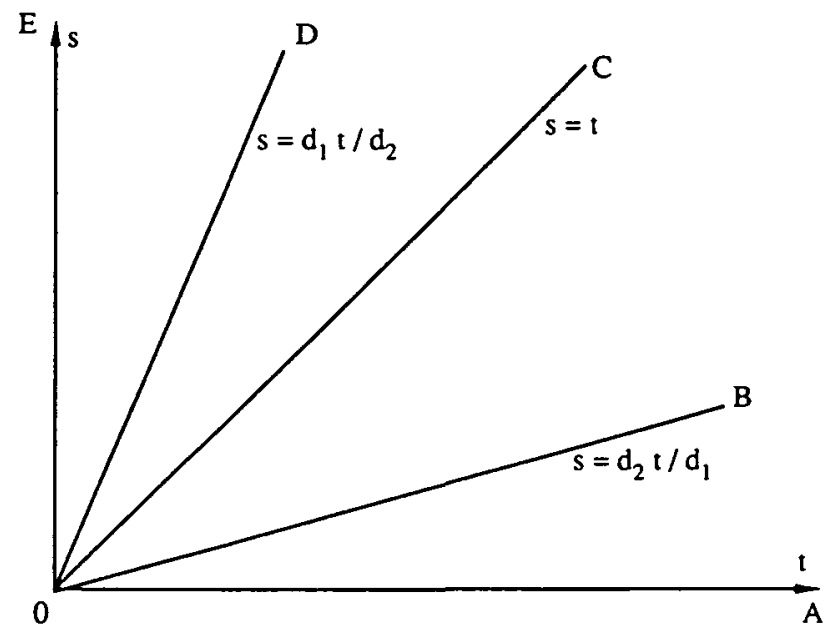

Figure 1 Characteristics for (2.10) and (2.13) for two dependent variables $c_{1}$ and $c_{2}$ satisfying (1.2) with $d_{1}>d_{2}>0$. 


$$
k_{21}^{*+}(t, t)=\frac{a_{21} e^{a_{11} t}}{\left(1-d_{2} / d_{1}\right)}
$$

Thus $k_{11}^{*+}(t, s)$ and $k_{21}^{*+}(t, s)$ are obtained by solving (2.10) subject to $(2.11)_{3,4}$ and (2.12) which we accomplish in the following section.

Similarly the second column of the kernel matrix $k^{*}(t, s)$ satisfies

$$
\begin{aligned}
\frac{\partial k_{12}^{*}}{\partial t}+\frac{d_{1}}{d_{2}} \frac{\partial k_{12}^{*}}{\partial s} & =a_{11} k_{12}^{*}+a_{12} k_{22}^{*}, \\
\frac{\partial k_{22}^{*}}{\partial t}+\frac{\partial k_{22}^{*}}{\partial s} & =a_{21} k_{12}^{*}+a_{22} k_{22}^{*},
\end{aligned}
$$

with boundary conditions

$$
\begin{gathered}
k_{12}^{*-}(0, s)=k_{22}^{*-}(0, s)=0 \\
k_{12}^{*+}(t, 0)=-a_{12} d_{2} / d_{1}, k_{22}^{*+}(t, 0)=-a_{22} .
\end{gathered}
$$

In this case $k_{12}^{*-}(t, s)$ and $k_{22}^{*-}(t, s)$ are zero in the region EOD. From these preliminary observations we may deduce from $(2.3)_{2}$

$$
\begin{aligned}
c_{1}(\underset{\sim}{x}, t)= & e^{a_{11} t} H_{1}(\underset{\sim}{x}, t)+\int_{t}^{d_{1} t / d_{2}} k_{12}^{*-}(t, s) H_{2}(\underset{\sim}{x}, s) d s \\
& +\int_{0}^{t}\left\{k_{11}^{*+}(t, s) H_{1}(\underset{\sim}{x}, s)+k_{12}^{*+}(t, s) H_{2}(\underset{\sim}{x}, s)\right\} d s \\
c_{2}(\underset{\sim}{x}, t)= & e^{a_{22} t} H_{2}(\underset{\sim}{x}, t)+\int_{t}^{d_{1} t / k_{2}} k_{22}^{*-}(t, s) H_{2}(\underset{\sim}{x}, s) d s \\
& +\int_{0}^{t}\left\{k_{21}^{*+}(t, s) H_{1}(\underset{\sim}{x}, s)+k_{22}^{*+}(t, s) H_{2} \underset{\sim}{\underset{\sim}{x}, s)\} d s}\right.
\end{aligned}
$$

which gives the general form of the uncoupling transformation. The details for the determination of the components of the kernel matrix $k^{*}(t, s)$ are given in the following two sections.

\section{SOLUTION FOR $k_{11}^{*}(t, s)$ AND $k_{21}^{*}(t, s)$}

With reference to Figure 1, we look for a solution in the region AOB which is a function of $s$ only, that is

$$
k_{11}^{*+}(t, s)=k_{1}(s), k_{21}^{*+}(t, s)=k_{2}(s)
$$


where $k_{1}(s)$ and $k_{2}(s)$ satisfy

$$
\frac{d k_{1}}{d s}=a_{11} k_{1}+a_{12} k_{2}, \frac{d k_{2}}{d s}=\frac{d_{1}}{d_{2}}\left(a_{21} k_{1}+a_{22} k_{2}\right),
$$

and are subject to the initial conditions

$$
k_{1}(0)=-a_{11}, \quad k_{2}(0)=-a_{21} d_{1} / d_{2}
$$

In a routine fashion we may deduce

$$
k_{1}(s)=A_{1} e^{\alpha_{1} *}+A_{2} e^{\alpha_{2}}, k_{2}(s)=B_{1} e^{\alpha_{1}} \cdot+B_{2} e^{\alpha_{2} e},
$$

where $\alpha_{1}$ and $\alpha_{2}$ are given by

$$
\begin{aligned}
& \alpha_{1}=\left(2 d_{2}\right)^{-1}\left\{\left(a_{11} d_{2}+a_{22} d_{1}\right)+\left[\left(a_{11} d_{2}-a_{22} d_{1}\right)^{2}+4 d_{1} d_{2} a_{12} a_{21}\right]^{1 / 2}\right\}, \\
& \alpha_{2}=\left(2 d_{2}\right)^{-1}\left\{\left(a_{11} d_{2}+a_{22} d_{1}\right)-\left[\left(a_{11} d_{2}-a_{22} d_{1}\right)^{2}+4 d_{1} d_{2} a_{12} a_{21}\right]^{1 / 2}\right\},
\end{aligned}
$$

and the constants $A_{1}, A_{2}, B_{1}$ and $B_{2}$ are as follows,

$$
\begin{aligned}
& A_{1}=\Delta^{-1}\left\{a_{11} d_{2} \alpha_{2}-\left(a_{11}^{2} d_{2}+a_{21} a_{12} d_{1}\right)\right\} \\
& A_{2}=-\Delta^{-1}\left\{a_{11} d_{2} \alpha_{1}-\left(a_{11}^{2} d_{2}+a_{21} a_{12} d_{1}\right)\right\} \\
& B_{1}=-\Delta^{-1} a_{21} d_{1} \alpha_{1}, B_{2}=\Delta^{-1} a_{21} d_{1} \alpha_{2},
\end{aligned}
$$

where $\Delta$ denotes the square root appearing in (3.5), namely

$$
\Delta=\left[\left(a_{11} d_{2}-a_{22} d_{1}\right)^{2}+4 d_{1} d_{2} a_{12} a_{21}\right]^{1 / 2},
$$

which we assume to be non-zero.

In order to solve (2.10) in the region BOC we observe that $k_{21}^{*+}(t, s)$ suffers a jump across $s=d_{2} t / d_{1}$ while $k_{11}^{*+}(t, s)$ is continuous across this line. Further it can be seen after a little thought that the natural variables of this problem are

$$
x=\frac{s-d_{2} t / d_{1}}{1-d_{2} / d_{1}}, y=\frac{t-s}{1-d_{2} / d_{1}}
$$

because on the line $s=d_{2} t / d_{1}$ we have $x=0, y=t$ while on the line $s=t$ we have $x=t, y=0$. Moreover on making the transformation

$$
k_{11}^{*+}=e^{a_{11} x+a_{22} y} \Psi_{11}(x, y), \quad k_{21}^{*+}=e^{a_{11} x+a_{22} y} \Psi_{21}(x, y),
$$

we find from (2.10) and (2.12) that we have

$$
\begin{gathered}
\frac{\partial \Psi_{11}}{\partial x}=a_{12} \Psi_{21}, \frac{\partial \Psi_{21}}{\partial y}=a_{21} \Psi_{11} \\
\Psi_{11}(0, y)=\Psi(y), \Psi_{21}(x, 0)=\frac{a_{21}}{\left(1-d_{2} / d_{1}\right)},
\end{gathered}
$$


where the function $\Psi(y)$ is known and is determined from $(3.4)_{1}$ and $(3.9)_{1}$, thus

$$
\Psi(y)=e^{-\alpha_{22} y}\left(A_{1} e^{\alpha_{1} d_{2} y / d_{1}}+A_{2} e^{\alpha_{2} d_{2} y / d_{1}}\right) .
$$

In the following it is convenient to introduce $\beta_{1}$ and $\beta_{2}$ defined by

$$
\beta_{1}=\left(\alpha_{1} d_{2}-a_{22} d_{1}\right) / d_{1}, \beta_{2}=\left(\alpha_{2} d_{2}-a_{22} d_{1}\right) / d_{1},
$$

so that the function $\Psi(y)$ becomes simply

$$
\Psi(y)=A_{1} e^{\beta_{1} y}+A_{2} e^{\beta_{2} y} .
$$

To obtain the solution we use the Laplace transform defined by

$$
\widehat{f}(p)=\int_{0}^{\infty} e^{-p y} f(y) d y,
$$

and from (3.10) and (3.13) we obtain the following equations for the Laplace transforms $\widehat{\Psi}_{11}(x, p)$ and $\widehat{\Psi}_{21}(x, p)$,

$$
\begin{gathered}
\frac{\partial \widehat{\Psi}_{11}}{\partial x}=a_{12} \widehat{\Psi}_{21}, \quad \widehat{\Psi}_{21}=\frac{a_{21}}{p}\left\{\widehat{\Psi}_{11}+\frac{1}{\left(1-d_{2} / d_{1}\right)}\right\}, \\
\widehat{\Psi}_{11}(0, y)=\frac{A_{1}}{\left(p-\beta_{1}\right)}+\frac{A_{2}}{\left(p-\beta_{2}\right)},
\end{gathered}
$$

which can be solved in a routine manner to yield

$$
\begin{aligned}
& \widehat{\Psi}_{11}(x, p)=\left\{\frac{A_{1}}{\left(p-\beta_{1}\right)}+\frac{A_{2}}{\left(p-\beta_{2}\right)}+\frac{1}{\left(1-d_{2} / d_{1}\right)}\right\} e^{a_{12} a_{21} x / p}-\frac{1}{\left(1-d_{2} / d_{1}\right)}, \\
& \widehat{\Psi}_{21}(x, p)=\frac{a_{21}}{p}\left\{\frac{A_{1}}{\left(p-\beta_{1}\right)}+\frac{A_{2}}{\left(p-\beta_{2}\right)}+\frac{1}{\left(1-d_{2} / d_{1}\right)}\right\} e^{a_{12} a_{21} x / p} .
\end{aligned}
$$

On making use of the non-trivial identity

$$
\frac{A_{1}}{\beta_{1}}+\frac{A_{2}}{\beta_{2}}=-\frac{d_{1}}{d_{2}}
$$

we can simplify the expression for $\widehat{\Psi}_{21}(x, p)$ to obtain

$$
\widehat{\Psi}_{21}(x, p)=a_{21}\left\{\frac{A_{1}}{\beta_{1}\left(p-\beta_{1}\right)}+\frac{A_{2}}{\beta_{2}\left(p-\beta_{2}\right)}+\frac{d_{1}}{p d_{2}\left(1-d_{2} / d_{1}\right)}\right\} e^{a_{12} a_{21} x / p} .
$$


On using equations (A1) - (A3) in the Appendix we can from (3.16) ${ }_{1}$ and (3.18) immediately deduce the following expressions

$$
\begin{aligned}
\widehat{\Psi}_{11}(x, y)= & A_{1} \phi\left(\beta_{1} y, a_{12} a_{21} x y\right)+A_{2} \phi\left(\beta_{2} y, a_{12} a_{21} x y\right) \\
& +\left(\frac{a_{12} a_{21} x}{y}\right)^{1 / 2} \frac{I_{1}\left[2\left(a_{12} a_{21} x y\right)^{1 / 2}\right]}{\left(1-d_{2} / d_{1}\right)}, \\
\widehat{\Psi}_{21}(x, y)= & \frac{a_{21} A_{1}}{\beta_{1}} \phi\left(\beta_{1} y, a_{12} a_{21} x y\right)+\frac{a_{21} A_{2}}{\beta_{2}} \phi\left(\beta_{2} y, a_{12} a_{21} x y\right) \\
& +\frac{d_{1} a_{21}}{d_{2}\left(1-d_{2} / d_{1}\right)} I_{0}\left[2\left(a_{12} a_{21} x y\right)^{1 / 2}\right],
\end{aligned}
$$

where as noted in the Appendix $\phi(x, y)$ denotes the hypergeometric function of two variables usually denoted by $\Phi_{3}(1,1 ; x, y)$ and for which various properties are noted in the Appendix.

\section{SOLUTION FOR $k_{12}^{*}(t, s)$ AND $k_{22}^{*}(t, s)$}

In the region $\mathrm{AOC}$ we look for a solution which is a function of $s$ only, namely

$$
k_{12}^{*+}(t, s)=\ell_{1}(s), \quad k_{22}^{*+}(t, s)=\ell_{2}(s),
$$

where $\ell_{1}(s)$ and $\ell_{2}(s)$ satisfy

$$
\frac{d \ell_{1}}{d s}=\frac{d_{2}}{d_{1}}\left(a_{11} \ell_{1}+a_{12} \ell_{2}\right), \frac{d \ell_{2}}{d s}=a_{21} \ell_{1}+a_{22} \ell_{2},
$$

and are subject to the initial conditions

$$
\ell_{1}(0)=-a_{12} d_{2} / d_{1}, \ell_{2}(0)=-a_{22} .
$$

Again in a routine manner we may deduce

$$
\ell_{1}(s)=C_{1} e^{\gamma_{1} \cdot}+C_{2} e^{\gamma_{2} \bullet}, \ell_{2}(s)=D_{1} e^{\gamma_{1} \cdot}+D_{2} e^{\gamma_{2} e},
$$

where $\gamma_{1}$ and $\gamma_{2}$ are given by

$$
\gamma_{1}=d_{2} \alpha_{1} / d_{1}, \gamma_{2}=d_{2} \alpha_{2} / d_{1},
$$

and the constants $C_{1}, C_{2}, D_{1}$ and $D_{2}$ are as follows,

$$
\begin{aligned}
& C_{1}=d_{2}\left(\Delta d_{1}\right)^{-1}\left\{a_{21} d_{2} \alpha_{2}-\left(a_{11} a_{21} d_{2}+a_{12} a_{22} d_{1}\right)\right\}, \\
& C_{2}=-d_{2}\left(\Delta d_{1}\right)^{-1}\left\{a_{21} d_{2} \alpha_{1}-\left(a_{11} a_{21} d_{2}+a_{12} a_{22} d_{1}\right)\right\}, \\
& D_{1}=\Delta^{-1}\left\{a_{22} d_{2} \alpha_{2}-\left(a_{22}^{2} d_{1}+a_{21}^{2} d_{2}\right)\right\} \\
& D_{2}=-\Delta^{-1}\left\{a_{22} d_{2} \alpha_{1}-\left(a_{22}^{2} d_{1}+a_{21}^{2} d_{2}\right)\right\}
\end{aligned}
$$


where $\alpha_{1}, \alpha_{2}$ and $\Delta$ are all as previously defined.

In the region $C O D$ we employ coordinates

$$
X=\frac{s-t}{\left(d_{1} / d_{2}-1\right)}, Y=\frac{d_{1} t / d_{2}-s}{\left(d_{1} / d_{2}-1\right)}
$$

so that on $s=t$ we have $X=0$ and $Y=t$ while on $s=d_{1} t / d_{2}$ we have $X=t$ and $Y=0$. On making the transformation

$$
k_{12}^{*-}=e^{a_{11} X+a_{22} Y} \Psi_{12}(X, Y), \quad k_{22}^{*-}=e^{a_{11} X+a_{22} Y} \Psi_{22}(X, Y),
$$

we find from (2.13), (2.8) and (4.4) $)_{1}$ that we have

$$
\begin{aligned}
& \frac{\partial \Psi_{12}}{\partial X}=a_{12} \Psi_{22}, \frac{\partial \Psi_{22}}{\partial Y}=a_{21} \Psi_{12}, \\
& \Psi_{12}(0, Y)=\Psi(Y), \Psi_{22}(X, 0)=0,
\end{aligned}
$$

where the function $\Psi(Y)$ is defined by

$$
\Psi(Y)=C_{1} e^{\beta_{1} Y}+C_{2} e^{\beta_{2} Y}+\frac{a_{12}}{\left(d_{1} / d_{2}-1\right)},
$$

where $\beta_{1}$ and $\beta_{2}$ are exactly as previously defined by (3.12). As before using the Laplace transform it is not difficult to show that

$$
\begin{aligned}
& \widehat{\Psi}_{12}(X, p)=\left\{\frac{C_{1}}{\left(p-\beta_{1}\right)}+\frac{C_{2}}{\left(p-\beta_{2}\right)}+\frac{a_{12}}{p\left(d_{1} / d_{2}-1\right)}\right\} e^{a_{12} a_{21} X / p}, \\
& \widehat{\Psi}_{22}(X, p)=\frac{a_{21}}{p}\left\{\frac{C_{1}}{\left(p-\beta_{1}\right)}+\frac{C_{2}}{\left(p-\beta_{2}\right)}+\frac{a_{12}}{p\left(d_{1} / d_{2}-1\right)}\right\} e^{a_{12} a_{21} X / p} .
\end{aligned}
$$

On making use of the identity

$$
\frac{C_{1}}{\beta_{1}}+\frac{C_{2}}{\beta_{2}}=-\frac{a_{22}}{a_{21}}
$$

we may deduce the following

$$
\begin{aligned}
\Psi_{12}(X, Y)= & C_{1} \phi\left(\beta_{1} Y, a_{12} a_{21} X Y\right)+C_{2} \phi\left(\beta_{2} Y, a_{12} a_{21} X Y\right) \\
& +\frac{a_{12}}{\left(d_{1} / d_{2}-1\right)} I_{0}\left[2\left(a_{12} a_{21} X Y\right)^{1 / 2}\right] \\
\Psi_{22}(X, Y)= & \frac{a_{21}}{\beta_{1}} C_{1} \phi\left(\beta_{1} Y, a_{12} a_{21} X Y\right)+\frac{a_{21} C_{2}}{\beta_{2}} \phi\left(\beta_{2} Y, a_{12} a_{21} X Y\right) \\
& +a_{22} I_{0}\left[2\left(a_{12} a_{21} X Y\right)^{1 / 2}\right]+\left(\frac{a_{12} a_{21} Y}{X}\right)^{1 / 2} \frac{I_{1}\left[2\left(a_{12} a_{21} X Y\right)^{1 / 2}\right]}{\left(d_{1} / d_{2}-1\right)}
\end{aligned}
$$

where on this occasion as well as using (A1) - (A3) we have made use of the Laplace transform arising from differentiating (A1) with respect to the parameter $\alpha$. 


\section{Summary OF RESUlts}

From the formulae of the previous two sections we can show directly from (2.15), after some considerable rearrangement, that in terms of $h_{1}(\underset{\sim}{x}, t)$ and $h_{2}(\underset{\sim}{x}, t)$ satisfying (1.6), the Dankwerts' transformation appropriate to the coupled system (1.2) becomes

$$
\begin{aligned}
& c_{1}(\underset{\sim}{x}, t)=e^{a_{11} t} h_{1}\left(\underset{\sim}{x}, d_{1} t\right) \\
& +\int_{0}^{d_{2} t}\left\{\left(A_{1} e^{\lambda_{1} \xi}+A_{2} e^{\lambda_{2} \xi}\right) \frac{h_{1}(\underset{\sim}{x}, \xi)}{d_{1}}+\left(C_{1} e^{\lambda_{1} \xi}+C_{2} e^{\lambda_{2} \xi}\right) \frac{h_{2}(\underset{\sim}{x}, \xi)}{d_{2}}\right\} d \xi \\
& +e^{\lambda t} \int_{d_{2} t}^{d_{1} t} e^{-\mu \xi}\left\{\frac{A_{1}}{d_{1}} \phi\left(\frac{\beta_{1}\left(d_{1} t-\xi\right)}{\left(d_{1}-d_{2}\right)}, \frac{\eta^{2}}{4}\right)+\frac{A_{2}}{d_{1}} \phi\left(\frac{\beta_{2}\left(d_{1} t-\xi\right)}{\left(d_{1}-d_{2}\right)}, \frac{\eta^{2}}{4}\right)\right. \\
& \left.+\left(\frac{a_{12} a_{21}\left(\xi-d_{2} t\right)}{\left(d_{1} t-\xi\right)}\right)^{1 / 2} \frac{I_{1}(\eta)}{\left(d_{1}-d_{2}\right)}\right\} h_{1}(\underset{\sim}{x}, \xi) d \xi \\
& +e^{\lambda t} \int_{d_{2^{t}}}^{d_{1} t} e^{-\mu \xi}\left\{\frac{C_{1}}{d_{2}} \phi\left(\frac{\beta_{1}\left(d_{1} t-\xi\right)}{\left(d_{1}-d_{2}\right)}, \frac{\eta^{2}}{4}\right)+\frac{C_{2}}{d_{2}}\left(\frac{\beta_{2}\left(d_{1} t-\xi\right)}{\left(d_{1}-d_{2}\right)}, \frac{\eta^{2}}{4}\right)\right. \\
& \left.+\frac{a_{12} I_{0}(\eta)}{\left(d_{1}-d_{2}\right)}\right\} h_{2}(\underset{\sim}{x}, \xi) d \xi \text {, } \\
& c_{2}(\underset{\sim}{x}, t)=e^{a_{22} t} h_{2}\left(\underset{\sim}{x}, d_{2} t\right) \\
& +\int_{0}^{d_{2} t}\left\{\left(B_{1} e^{\lambda_{1} \xi}+B_{2} e^{\lambda_{2} \xi}\right) \frac{h_{1}(\underset{\sim}{x}, \xi)}{d_{1}}+\left(D_{1} e^{\lambda_{1} \xi}+D_{2} e^{\lambda_{2} \xi}\right) \frac{h_{2}(\underset{\sim}{x}, \xi)}{d_{2}}\right\} d \xi \\
& +e^{\lambda t} \int_{d_{2} t}^{d_{1} t} e^{-\mu t}\left\{\frac{B_{1}}{d_{1}} \phi\left(\frac{\beta_{1}\left(d_{1} t-\xi\right)}{\left(d_{1}-d_{2}\right)}, \frac{\eta^{2}}{4}\right)+\frac{B_{2}}{d_{1}} \phi\left(\frac{\beta_{2}\left(d_{1} t-\xi\right)}{\left(d_{1}-d_{2}\right)}, \frac{\eta^{2}}{4}\right)\right. \\
& \left.+\frac{d_{1} a_{21}}{d_{2}\left(d_{1}-d_{2}\right)} I_{0}(\eta)\right\} h_{1}(\underset{\sim}{x}, \xi) d \xi \\
& +e^{\lambda t} \int_{d_{2} t}^{d_{1} t} e^{-\mu \xi}\left\{\frac{D_{1}}{d_{2}} \phi\left(\frac{\beta_{1}\left(d_{1} t-\xi\right)}{\left(d_{1}-d_{2}\right)}, \frac{\eta^{2}}{4}\right)+\frac{D_{2}}{d_{2}} \phi\left(\frac{\beta_{2}\left(d_{1} t-\xi\right)}{\left(d_{1}-d_{2}\right)}, \frac{\eta^{2}}{4}\right)\right. \\
& \left.+\frac{a_{22}}{d_{2}} I_{0}(\eta)+\left(\frac{a_{12} a_{21}\left(d_{1} t-\xi\right)}{\left(\xi-d_{2} t\right)}\right)^{1 / 2} \frac{I_{1}(\eta)}{\left(d_{1}-d_{2}\right)}\right\} h_{2}(\underset{\sim}{x}, \xi) d \xi,
\end{aligned}
$$

where $\lambda, \mu$ and $\eta$ are defined by (1.4) and (1.5) while the $A_{j}, B_{j}, C_{j}, D_{j}$ and $\beta_{j}(j=1,2)$ are defined by equations (3.6), (4.6) and (3.12) respectively. Further the constants $\lambda_{j}$ are defined in terms of $\alpha_{j}$ and $\gamma_{j}(j=1,2)$ as follows,

$$
\lambda_{j}=\frac{\alpha_{j}}{d_{1}}=\frac{\gamma_{j}}{d_{2}}(j=1,2),
$$


where $\alpha_{j}$ and $\gamma_{j}$ are defined by (3.5) and (4.5) respectively.

In deriving (5.1) we have made frequent use of the important relations

$$
\beta_{1} \beta_{2}=-\frac{d_{2}}{d_{1}} a_{12} a_{21}, \quad a_{21} \frac{A_{j}}{\beta_{j}}=B_{j}, a_{21} \frac{C_{j}}{\beta_{j}}=D_{j}(j=1,2),
$$

which can be verified by elementary processes. We observe that the two formulae of equation (5.1) can be seen to be symmetric counterparts by making use of the identity $(A 8)_{3}$ and the important relations (3.17) and (4.12). Thus we may confirm that an alternative expression for $c_{2}(\underset{\sim}{x}, t)$ is as follows,

$$
\begin{aligned}
& c_{2}(\underset{\sim}{x}, t)=e^{a_{22} t} h_{2}\left(\underset{\sim}{x}, d_{2} t\right) \\
& +\int_{0}^{d_{1} t}\left\{\left(B_{1} e^{\lambda_{1} \xi}+B_{2} e^{\lambda_{2} \xi}\right) \frac{h_{1}(\underset{\sim}{x}, \xi)}{d_{1}}+\left(D_{1} e^{\lambda_{1} \xi}+D_{2} e^{\lambda_{2} \xi}\right) \frac{h_{2}(\underset{\sim}{x}, \xi)}{d_{2}}\right\} d \xi \\
& -e^{\lambda t} \int_{d_{2} t}^{d_{1} t} e^{-\mu \xi}\left\{\frac{B_{1}}{d_{1}} \phi\left(-\frac{\beta_{2} d_{1}\left(\xi-d_{2} t\right)}{d_{2}\left(d_{1}-d_{2}\right)}, \frac{\eta^{2}}{4}\right)+\frac{B_{2}}{d_{1}} \phi\left(-\frac{\beta_{1} d_{1}\left(\xi-d_{2} t\right)}{d_{2}\left(d_{1}-d_{2}\right)}, \frac{\eta^{2}}{4}\right)\right. \\
& \left.-\frac{a_{21} I_{0}(\eta)}{\left(d_{1}-d_{2}\right)}\right\} h_{1}(\underset{\sim}{x}, \xi) d \xi \\
& -e^{\lambda t} \int_{d_{2} t}^{d_{1} t} e^{-\mu \xi}\left\{\frac{D_{1}}{d_{2}} \phi\left(-\frac{\beta_{2} d_{1}\left(\xi-d_{2} t\right)}{d_{2}\left(d_{1}-d_{2}\right)}, \frac{\eta^{2}}{4}\right)+\frac{D_{2}}{d_{2}} \phi\left(-\frac{\beta_{1} d_{1}\left(\xi-d_{2} t\right)}{d_{2}\left(d_{1}-d_{2}\right)}, \frac{\eta^{2}}{4}\right)\right. \\
& \left.\left.-\left(\frac{a_{12} a_{21}\left(d_{1} t-\xi\right)}{\left(\xi-d_{2} t\right)}\right)^{1 / 2} \frac{I_{1}(\eta)}{\left(d_{1}-d_{2}\right)}\right\} h_{2} \underset{\sim}{x}, \xi\right) d \xi .
\end{aligned}
$$

The importance of these formulae, together with (1.3), is that solutions of boundary and initial value problems for the coupled system (1.1) which involve constant boundary data, can now be decomposed in a manner which is commonly exploited for solutions of boundary and initial value problems for the classical heat equation. That is, we can view such solutions of (1.1) as consisting of two contributions, one which is zero on the boundary with a non-zero initial condition and one with zero initial data but non-zero constant boundary data. Further the existence of these results also means that the substantial body of information known for the classical heat equation can now be exploited for coupled systems. Evidently however, for specific problems, such formulae need to be supplemented with standard integration routines and one advantage in using the procedure recommended here is that, having set up the integration codes for one problem, the same codes can be used for other problems by simply feeding in the appropriate classical heat functions $h_{1}(\underset{\sim}{x}, t)$ and $h_{2}(\underset{\sim}{x}, t)$. 


\section{APPENDIX}

For convenience we note in this appendix, the Laplace transforms and certain properties of the hypergeometric function of two variables $\Phi_{3}(1,1 ; x, y)$ and its relation to the $J$-function. The first two are standard (see for example, Oberhettinger and Badii [7]), namely

$$
\begin{aligned}
& \int_{0}^{\infty} e^{-p t} I_{0}\left[2(\alpha t)^{1 / 2}\right] d t=\frac{e^{\alpha / p}}{p}, \\
& \int_{0}^{\infty} e^{-p t}\left(\frac{\alpha}{t}\right)^{1 / 2} I_{1}\left[2(\alpha t)^{1 / 2}\right] d t=e^{\alpha / p}-1,
\end{aligned}
$$

for $\alpha>0$. The third transform which can be readily verified is

$$
\int_{0}^{\infty} e^{-p t} \phi(\beta t, \alpha t) d t=\frac{e^{\alpha / p}}{p-\beta}
$$

for $\alpha, \beta>0$ where $\phi(x, y)$ is the notation used throughout this paper for $\Phi_{3}(1,1 ; x, y)$, thus

$$
\phi(x, y)=\Phi_{3}(1,1 ; x, y)=\sum_{m, n=0}^{\infty} \frac{x^{m} y^{n}}{(m+n) ! n !}=e^{y / x} \int_{y / x}^{\infty} e^{-\xi} I_{0}\left[2(x \xi)^{1 / 2}\right] d \xi
$$

The $J$-function is usually defined by the equation (see for example Luke [4])

$$
J(x, y)=1-e^{-y} \int_{0}^{x} e^{-\xi} I_{0}\left[2(y \xi)^{1 / 2}\right] d \xi,
$$

so that from (A1) we have

$$
J(x, y)=e^{-y} \int_{x}^{\infty} e^{-\xi} I_{0}\left[2(y \xi)^{1 / 2}\right] d \xi,
$$

and from this equation and the last equality of (A4) we see that

$$
\phi(x, y)=e^{x+y / x} J(y / x, x) .
$$

The transform (A3) follows since

$$
\begin{aligned}
\int_{0}^{\infty} e^{-p t} \phi(\beta t, \alpha t) d t & =\sum_{m, n=0}^{\infty} \frac{\beta^{m} \alpha^{n}}{(m+n) ! n !} \int_{0}^{\infty} e^{-p t} t^{m+n} d t \\
& =\frac{1}{p} \sum_{m, n=0}^{\infty}\left(\frac{\beta}{p}\right)^{m}\left(\frac{\alpha}{p}\right)^{n} \frac{1}{n !}=\frac{e^{\alpha / p}}{p(1-\beta / p)}
\end{aligned}
$$


from which the desired result follows, assuming $|\beta / p|<1$ and that the formal manipulations can be justified. The last equality of (A4) can be most easily verified as follows,

$$
\begin{aligned}
e^{y / x} \int_{y / x}^{\infty} e^{-\xi} I_{0}\left[2(x \xi)^{1 / 2}\right] d \xi & =\int_{z}^{\infty} e^{-(\xi-z)} I_{0}\left[2(x \xi)^{1 / 2}\right] d \xi \\
& =\int_{0}^{\infty} e^{-u} I_{0}\left[2 x^{1 / 2}(u+z)^{1 / 2}\right] d u
\end{aligned}
$$

where $z=y / x$ and we have made the substitution $u=\xi-z$. Now the last integral becomes

$$
\begin{gathered}
\int_{0}^{\infty} \sum_{k=0}^{\infty} e^{-u} \frac{x^{k}(u+z)^{k}}{(k !)^{2}} d u=\int_{0}^{\infty} \sum_{k=0}^{\infty} \sum_{m=0}^{k} \frac{e^{-u} x^{k} u^{m} z^{k-m}}{k ! m !(k-m) !} d u \\
=\sum_{k=0}^{\infty} \sum_{m=0}^{k} \frac{x^{k} z^{k-m}}{k !(k-m) !}=\sum_{m=0}^{\infty} \sum_{k=m}^{\infty} \frac{x^{k} z^{k-m}}{k !(k-m) !}
\end{gathered}
$$

and $n=k-m$ gives the desired result. Funally we note the elementary properties of $\phi(x, y)$,

$$
\begin{gathered}
\phi(0, y)=I_{0}\left(2 y^{1 / 2}\right), \phi(x, 0)=e^{x} \\
\phi(x, y)+\phi(y / x, y)=I_{0}\left(2 y^{1 / 2}\right)+e^{x+y / x}
\end{gathered}
$$

The first two follow immediately from the series for $\phi(x, y)$. For the third result, with $z=y / x$ we have

$$
\begin{gathered}
\phi(x, y)+\phi(y / x, y)=\sum_{k=0}^{\infty} \sum_{n=0}^{k}\left(\frac{x^{k}}{k !} \frac{z^{n}}{n !}+\frac{z^{k}}{k !} \frac{x^{n}}{n !}\right) \\
=\sum_{k=0}^{\infty} \sum_{n=0}^{k} \frac{x^{k}}{k !} \frac{z^{n}}{n !}+\sum_{n=0}^{\infty} \sum_{k=n}^{\infty} \frac{z^{k}}{k !} \frac{x^{n}}{n !}
\end{gathered}
$$

and the final double series becomes

$$
\begin{gathered}
\sum_{n=0}^{\infty} \sum_{k=0}^{\infty} \frac{z^{k}}{k !} \frac{x^{n}}{n !}-\sum_{n=0}^{\infty} \sum_{k=0}^{n-1} \frac{z^{k}}{k !} \frac{x^{n}}{n !} \\
=e^{x+y / z}-\sum_{k=0}^{\infty} \sum_{n=0}^{k-1} \frac{z^{n}}{n !} \frac{x^{k}}{k !}
\end{gathered}
$$


so that altogether we have

$$
\phi(x, y)+\phi(y / x, y)=\sum_{k=0}^{\infty} \frac{(x y)^{k}}{(k !)^{2}}+e^{x+y / x},
$$

which immediately gives the desired result. In terms of the $J$-function the elementary properties (A8) are simply the standard formulae

$$
\begin{gathered}
J(x, 0)=e^{-x}, J(0, y)=1, \\
J(x, y)+J(y, x)=1+e^{-(x+y)} I_{0}\left[2(x y)^{1 / 2}\right],
\end{gathered}
$$

as can be readily confirmed.

\section{REFERENCES}

[1] J. Crank, The mathematics of diffusion, 2nd Ed. (Clarendon Press, Oxford, 1975).

[2] J.M. Hill, 'On the solution of reaction-diffusion equations', IMA J. Appl. Math. 27 (1981), 177-194.

[3] J.M. Hill and A. McNabb, 'On the problem of uncoupling systems of linear differential equations', J. Austral. Math. Soc. Ser. B 30 (1989), 483-501.

[4] Y.L. Luke, Integrals of Bessel functions (McGraw-Hill, New York, 1962).

[5] A. McNabb, 'An uncoupling procedure for a class of coupled linear partial differential equations', J. Austral. Math. Soc. Ser B 26 (1985), 503-513.

[6] A. McNabb, 'A generalised Dankwerts' transformation'. (submitted) .

[7] F. Oberhettinger and L. Badii, 1973 Tables of Laplace transforms (Springer-Verlag, Berlin, Heidelberg, New York, 1973).

Department of Mathematics

University of Wollongong

Wollongong NSW 2500

Australia
Department of Mathematics and Statistics Massey University

Palmerston North

New Zealand 